\title{
Conhecimento atual sobre transmissão vertical de SARS-CoV-2: uma revisão de Iiteratura
}

\section{Current knowledge about vertical transmission of SARS- CoV-2: a literature review}

Beatriz Cavallari', Denise Cristiane da $\mathrm{Cruz}^{2}$, João Felipe Marques da Silva ${ }^{3}$, Camila Rodrigues Ferraz ${ }^{4}$

1.ORCID: https://orcid.org/0000-0001-5195-5390. Graduanda em enfermagem. Faculdade de Tecnologia do Vale do Ivaí, Ivaiporã, Paraná, Brasil.

E-mail: bcavallari123@gmail.com

2.ORCID:https://orcid.org/0000-0001-9209-2341. Graduanda em enfermagem. Faculdade de Tecnologia do Vale do Ivaí, Ivaiporã, Paraná, Brasil.

E-mail: denisecristiane999@gmail.com

3.ORCID: https://orcid.org/0000-0001-7198-8528. Mestre em Saúde Coletiva. Faculdade de Tecnologia do Vale do Ivaí, Ivaiporã, Paraná, Brasil.

E-mail: joaomarques371@gmail.com

4.ORCID: https://orcid.org/0000-0003-4550-0773. Doutora em Ciências da Saúde. Universidade Estadual de Londrina, Londrina, Paraná, Brasil.

E-mail: camila ferraz96@hotmail.com

CONTATO: Autor correspondente: Camila Rodrigues Ferraz | Endereço: Departamento de Ciências Patológicas, Universidade Estadual de Londrina, Rod. Celso Garcia Cid Km480 PR445, CEP 86057-970, Londrina, Paraná, Brasil. Telefone: (67) 981897262 E-mail: camila ferraz96@hotmail.com

\section{RESUMO}

Frente sua especificidade, nota-se a dimensão e a complexidade de Severe acute respiratory syndrome coronavirus no cenário atual. Até o momento, há dúvidas pertinentes sobre o potencial de contaminação nas gestantes e recém-nascidos, assim, por meio de uma revisão integrativa, o presente estudo teve como objetivo elencar os riscos eminentes à saúde desse grupo. 
Após a seleção dos artigos no período estabelecido (até setembro de 2020) utilizamos 37 artigos e 3 documentos oficiais para análise da literatura. Evidenciamos a eminente necessidade de compreender a relação de Severe acute respiratory syndrome coronavirus e as possíveis complicações desencadeadas por esse vírus no binômio mãe e filho. Em suma, há fortes indícios da existência da transmissão vertical de Severe acute respiratory syndrome coronavirus, assim, torna-se extremamente necessário a elaboração de cuidados assistenciais ao binômio mãe e filho, por intermédio da inserção de protocolos nacionais e adesão de cuidados básicos pertinentes à prevenção.

DESCRITORES: Infecções por Coronavírus. Saúde Materna. Transmissão Vertical de Doença Infecciosa. Gerenciamento Clínico.

\section{ABSTRACT}

The dimension and complexity of Severe acute respiratory syndrome coronavirus have become a major challenge for mankind and science and can trigger from sequels mild to lethal. So far, there are pertinent doubts about the potential for contamination in pregnant women and newborns, so, through an integrative review, the present study aimed to list the eminent health risks of this group that need extra attention, resulting from their existing immunological, physiological and physical weaknesses. After selecting the articles in the established period (articles published until September 2020), we included 37 articles and 3 official documents for literature analysis. In view of the pregnant woman's vulnerability and exposure to certain viruses that cause serious pathologies, there is a wide and distinct variety of complications that can affect the mother and child. Thus, there is an eminent need to understand the relationship between SARS-CoV-2 and the possible complications triggered by this virus in the pregnant woman's body. There is strong evidence of the existence of vertical transmission of SARS-CoV-2. Thus, it becomes relevant to observe and elaborate care provided to the mother and child, through the insertion of national protocols and adherence to basic care related to prevention.

DESCRIPTORS: Coronavirus infections. Maternal Health. Vertical Transmission of Infectious Disease. Clinical Management. 


\section{INTRODUÇÃO}

$\mathbf{N}$ o final de 2019, na cidade de Wuhan na China, foram identificados diversos casos de infecções respiratórias causadas por um novo vírus, altamente transmissível, denominado SARS-CoV-2 (Severe acute respiratory syndrome coronavirus) ${ }^{1}$. O Coronavírus pertence à família Coronaviridae. A propagação do vírus foi vertiginosa e seu principal meio de transmissão é entre pessoas por aerossóis ou após tocar em superfícies contaminadas, seguido de contato com os olhos, nariz ou boca $^{3}$. O surto foi declarado Emergência de Saúde Pública de Interesse Internacional, em 30 de janeiro de $2020^{4}$.

Em 11 de fevereiro de 2020, a Organização Mundial da Saúde (OMS) anunciou "COVID-19" (Coronavirus Disease 2019) como o nome desta nova doença. No Brasil, a COVID-19 foi declarada como emergência de saúde pública no dia 03 de fevereiro de 2020 e, no dia 06 de fevereiro, o Ministério da Saúde aprovou a Lei da Quarentena 5 . Entretanto, o primeiro caso confirmado foi em 26 de fevereiro, em São Paulo. No mesmo mês, começaram as primeiras ações governamentais ligadas à pandemia da COVID-19. A forma como ela se apresenta é ampla, variando desde assintomáticos até pacientes com manifestações graves até mesmo óbito ${ }^{3}$.

Grande parte das infecções pulmonares são de grau leve, contudo, formas graves também são descritas, especialmente na população considerada de risco, em que incluem os idosos e indivíduos com outras condições subjacentes e gestantes, podendo evoluir com falta de ar, diminuição da concentração de oxigênio no sangue, elevado comprometimento pulmonar nos estudos radiológicos, insuficiência respiratória, choque e falência múltipla de órgãos ${ }^{3}$.

Sabe-se que as mulheres grávidas são afetadas desproporcionalmente por doenças respiratórias, que estão associadas ao aumento da morbidade e altas taxas de mortalidade materna. A síndrome respiratória aguda (SARS-CoV) e síndrome respiratória do Oriente Médio (MERS-CoV) das últimas duas décadas foram especialmente graves, com aproximadamente um terço das mulheres grávidas infectadas e muitos casos óbito ${ }^{4,6}$.

Atualmente, o conhecimento sobre a COVID-19, particularmente sobre os seus efeitos em mulheres grávidas e recém-nascidos (RNs) é pequeno. Não há um 
consenso se o vírus SARS-CoV-2 pode ser transmitido verticalmente (transmissão durante a gestação, parto ou amamentação), mas é sabido que uma mãe infectada pode transmitir o vírus através de gotículas respiratórias durante a amamentação ${ }^{7}$.

Neste estudo, revisamos as características epidemiológicas e os aspectos clínicos de gestantes infectadas com SARS-CoV-2 até o momento. Também discutimos os achados perinatais de RNs de mulheres infectadas com SARS-CoV-2 durante a gravidez. A compreensão da apresentação clínica desse vírus em gestantes, torna-se importante para a identificação precoce de possíveis complicações do SARS-CoV-2, na formação fetal e saúde materna, além de auxiliar na elaboração de protocolos oficiais de manejo.

\section{MÉTODO}

Trata-se de uma revisão narrativa de literatura, dividida em duas etapas: (i) a revisão da literatura científica especializada, mediante busca na base de dados PubMed e SciELO; e (ii) a revisão de endereços on-line, relatórios e documentos oficiais nacionais e internacionais sobre a temática Covid-19 e gestação.

A busca na base de dados PubMed e SciELO foi realizada utilizando os seguintes descritores: COVID-19, SARS-CoV-2, pregnant. Foram incluídos trabalhos com relato de pelo menos uma evidencia clínica sobre a influência da Covid-19 na gestação. Houve restrição quanto ao idioma, somente artigos em português ou inglês e ao período de publicação, artigos publicados até setembro de 2020 e não houve restrição quanto ao desenho de estudo. Foram excluídos trabalhos que (i) não discutiam as implicações da Covid-19 na gestação (ii) não forneciam resumo/abstract e (iii) estavam em outros idiomas.

A busca resultou em 304 registros. Após rastreamento de título e resumo, e aplicação dos critérios de elegibilidade, foram selecionados 37 artigos para leitura do texto completo (Figura 1). Os artigos finais e os documentos oficiais selecionados foram analisados por dois autores, de maneira independente e aleatorizada. 
Figura 1. Fluxograma de seleção dos trabalhos para a revisão narrativa.

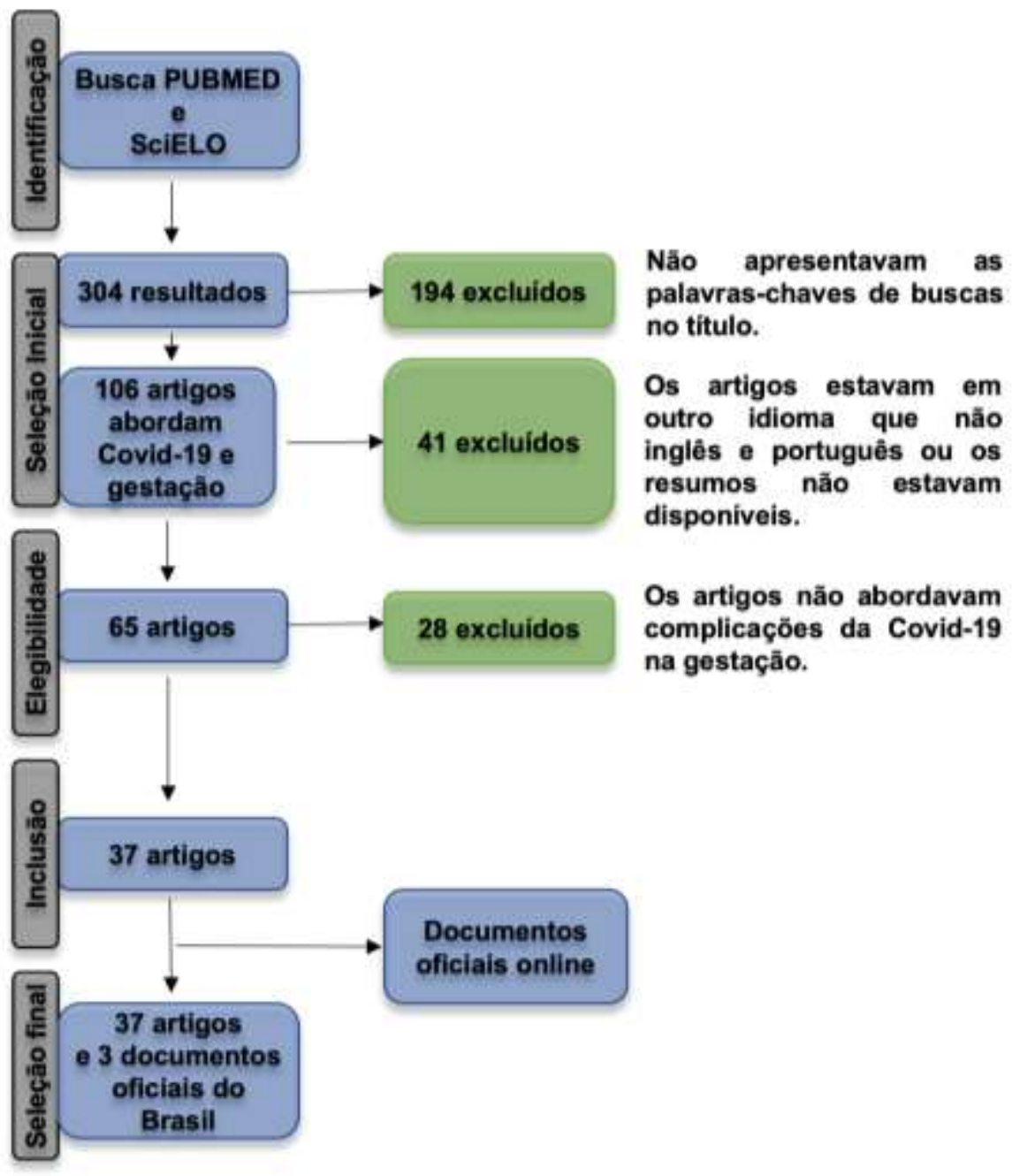

Fonte: Elaborado pelos autores (2021).

\section{RESULTADOS}

A partir dos artigos considerados elegíveis e das informações provenientes de outras fontes, foram identificadas três grupos de análise: Achados clínicos, manejo e condutas, e óbito materno.

O Quadro 1 e 2 mostram, em detalhes, os documentos oficiais e artigos selecionados.

Quadro 1. Organização de Documentos Oficiais e Centros Formadores

\begin{tabular}{|c|l|c|}
\hline \multicolumn{1}{|c|}{ AUTOR } & \multicolumn{1}{|c|}{ TíTULO REF } & \multicolumn{1}{|c|}{} \\
\hline Ministério da Saúde. & $\begin{array}{l}\text { Atenção à saúde do recém-nascido no } \\
\text { contexto da infecção pelo novo } \\
\text { coronavírus. }\end{array}$ & 39 \\
\hline
\end{tabular}




\begin{tabular}{|l|l|c|}
\hline Ministério da Saúde. & $\begin{array}{l}\text { Assistência à gestante e puérpera frente } \\
\text { à pandemia de COVID-19 }\end{array}$ & 45 \\
\hline $\begin{array}{l}\text { Secretaria da Saúde de } \\
\text { Tocantins. }\end{array}$ & $\begin{array}{l}\text { Atenção à Gestante - Coronavírus } \\
\text { (COVID-19). }\end{array}$ & 35 \\
\hline
\end{tabular}

Fonte: Elaborado pelos autores (2021).

Quadro 2. Organização de Artigos Indexados em bases internacionais

\begin{tabular}{|c|c|c|}
\hline AUTORES & TÍTULO & REF \\
\hline Alfaraj et al. & $\begin{array}{l}\text { "East Respiratory Syndrome Coronavirus (MERS-CoV) } \\
\text { infection during pregnancy: Report of two cases \& review } \\
\text { of the literature" }\end{array}$ & 6 \\
\hline $\begin{array}{l}\text { Borges do } \\
\text { Nascimento et } \\
\text { al. }\end{array}$ & $\begin{array}{l}\text { "Novel Coronavirus Infection (COVID-19) in Humans: A } \\
\text { Scoping Review and Meta-Analysis" }\end{array}$ & 1 \\
\hline Chate et al. & $\begin{array}{l}\text { "Presentation of pulmonary infection on CT in COVID-19: } \\
\text { initial experience in Brazil" }\end{array}$ & 3 \\
\hline Chen et al. & $\begin{array}{l}\text { "Expert consensus for managing pregnant women and } \\
\text { neonates born to mothers with suspected or confirmed } \\
\text { novel coronavirus (COVID-19) infection" }\end{array}$ & 36 \\
\hline Chen et al. & $\begin{array}{l}\text { "Clinical characteristics and intrauterine vertical } \\
\text { transmission potential of COVID-19 infection in nine } \\
\text { pregnant women: A retrospective review of medical } \\
\text { records" }\end{array}$ & 16 \\
\hline Chua et al. & $\begin{array}{l}\text { "From the frontline of COVID-19 - how prepared are we } \\
\text { as obstetricians? A commentary" }\end{array}$ & 37 \\
\hline Croda et al. & $\begin{array}{l}\text { "Covid-19 in Brazil: Advantages of a socialized unified } \\
\text { health system and preparation to contain cases" }\end{array}$ & 5 \\
\hline Dana et al. & $\begin{array}{l}\text { "COVID-19 and pregnancy: A Review of current } \\
\text { knowledge" }\end{array}$ & 13 \\
\hline Dashraath et al. & $\begin{array}{l}\text { "Coronavirus disease } 2019 \text { (COVID-19) pandemic and } \\
\text { pregnancy }\end{array}$ & 38 \\
\hline $\begin{array}{l}\text { De Seixas Filho } \\
\text { et al. }\end{array}$ & $\begin{array}{l}\text { Recomendações de Prevenção da Saúde Materno-Infantil } \\
\text { da Pandemia Covid-19 por meio de Protocolos Médicos }\end{array}$ & 40 \\
\hline
\end{tabular}




\begin{tabular}{|c|c|c|}
\hline De Sousa et al. & $\begin{array}{l}\text { "Effects of COVID-19 infection during pregnancy and } \\
\text { neonatal prognosis: What is the evidence?" }\end{array}$ & 29 \\
\hline Di Mascio et al. & $\begin{array}{l}\text { "Outcome of coronavirus spectrum infections (SARS, } \\
\text { MERS, COVID-19) during pregnancy: a systematic review } \\
\text { and meta-analysis" }\end{array}$ & 23 \\
\hline Ellington et al. & $\begin{array}{l}\text { "Characteristics of Women of Reproductive Age with } \\
\text { Laboratory-Confirmed SARS-CoV-2 Infection by } \\
\text { Pregnancy Status" }\end{array}$ & 43 \\
\hline Elshafeey et al. & $\begin{array}{l}\text { "A systematic scoping review of COVID-19 during } \\
\text { pregnancy and childbirth" }\end{array}$ & 18 \\
\hline Juan et al. & $\begin{array}{l}\text { "Effect of coronavirus disease } 2019 \text { (COVID-19) on } \\
\text { maternal, perinatal and neonatal outcome: Systematic } \\
\text { review" }\end{array}$ & 33 \\
\hline $\begin{array}{l}\text { Karimi-Zarchi } \\
\text { et al. }\end{array}$ & $\begin{array}{l}\text { "Vertical Transmission of Coronavirus Disease } 19 \text { (COVID- } \\
\text { 19) from Infected Pregnant Mothers to Neonates: A } \\
\text { Review" }\end{array}$ & 10 \\
\hline $\begin{array}{l}\text { Kasraeian et } \\
\text { al. }\end{array}$ & $\begin{array}{l}\text { "COVID-19 pneumonia and pregnancy: a systematic } \\
\text { review and meta-analysis" }\end{array}$ & 11 \\
\hline Knight et al. & $\begin{array}{l}\text { "Characteristics and outcomes of pregnant women } \\
\text { admitted to hospital with confirmed SARS-CoV-2 infection } \\
\text { in UK: National population based cohort study" }\end{array}$ & 42 \\
\hline $\begin{array}{l}\text { Liang } \\
\text { Acharya }\end{array}$ & $\begin{array}{l}\text { "Novel corona virus disease (COVID-19) in pregnancy: } \\
\text { What clinical recommendations to follow?" }\end{array}$ & 26 \\
\hline Liu et al. & $\begin{array}{l}\text { "Clinical manifestations and outcome of SARS-CoV-2 } \\
\text { infection during pregnancy" }\end{array}$ & 9 \\
\hline Lowe e Bopp & "COVID-19 vaginal delivery - A case report" & 22 \\
\hline $\begin{array}{l}\text { Martins-Filho, } \\
\text { Santos e } \\
\text { Santos }\end{array}$ & $\begin{array}{l}\text { "To breastfeed or not to breastfeed? Lck of evidence on } \\
\text { the presence of SARS-CoV-2 in breastmilk of pregnant } \\
\text { women with COVID-19" }\end{array}$ & 21 \\
\hline $\begin{array}{l}\text { Panahi, Amiri e } \\
\text { Pouy }\end{array}$ & $\begin{array}{l}\text { "Clinical Characteristics of COVID-19 Infection in } \\
\text { Newborns and Pediatrics: A Systematic Review" }\end{array}$ & 15 \\
\hline $\begin{array}{l}\text { Panahi, Amiri e } \\
\text { Pouy }\end{array}$ & $\begin{array}{l}\text { "Risks of Novel Coronavirus Disease (COVID-19) in } \\
\text { Pregnancy: a Narrative Review" }\end{array}$ & 34 \\
\hline
\end{tabular}




\begin{tabular}{|l|l|c|}
\hline Pereira et al. & "Clinical course of coronavirus disease-2019 in pregnancy" & 14 \\
\hline $\begin{array}{l}\text { Peyronnet et } \\
\text { al. }\end{array}$ & $\begin{array}{l}\text { "SARS-CoV-2 infection during pregnancy. Information and } \\
\text { proposal of management care" }\end{array}$ & 12 \\
\hline Qiao & $\begin{array}{l}\text { "What are the risks of COVID-19 infection in pregnant } \\
\text { women?" }\end{array}$ & 30 \\
\hline Rajewska et al. & "COVID-19 and pregnancy-where are we now? A review" & 17 \\
\hline $\begin{array}{l}\text { Rasmussen et } \\
\text { al. }\end{array}$ & $\begin{array}{l}\text { "Coronavirus Disease 2019 (COVID-19) and pregnancy: } \\
\text { what obstetricians need to know" }\end{array}$ & 7 \\
\hline Rondelli et al. & $\begin{array}{l}\text { Assistência às Gestantes e Recém-Nascidos no Contexto } \\
\text { da Infecção Covid-19: Uma Revisão Sistemática }\end{array}$ & 28 \\
\hline Schwartz & $\begin{array}{l}\text { "An analysis of 38 pregnant women with COVID-19, their } \\
\text { newborn infants, and maternal-fetal transmission of SARS- } \\
\text { CoV-2: Maternal coronavirus infections and pregnancy } \\
\text { outcomes" }\end{array}$ & 8 \\
\hline Shanes et al. & "Placental Pathology in COVID-19" & 27 \\
\hline Takemoto et al. & $\begin{array}{l}\text { "The tragedy of COVID-19 in Brazil: 124 maternal deaths } \\
\text { and counting" }\end{array}$ & 41 \\
\hline Yan et al. & $\begin{array}{l}\text { "Coronavirus disease 2019 in pregnant women: a report } \\
\text { based on 116 cases" }\end{array}$ & 24 \\
\hline Yang et al. & "Novel coronavirus infection and pregnanc" & $\begin{array}{l}25 \\
\text { pregnant patients with COVID-19 in Wuhan, China: a } \\
\text { retrospective, single-centre, descriptive study" }\end{array}$ \\
\hline Yu et al. & $\begin{array}{l}\text { "COVID-19 in Children, Pregnancy and Neonates: A } \\
\text { Review of Epidemiologic and Clinical Features" }\end{array}$ & 20 \\
\hline
\end{tabular}

\section{DISCUSSÃO}

\section{Achados clínicos}

Atualmente, há dados sugerindo fortemente a transmissão materno-fetal e neonatal do SARS-CoV-28-17, 46 .

Estudo conduzido por Elshafeey et al. 202018, constatou que de $256 \mathrm{RNs}$, quatro foram testados como positivos, o mesmo ocorreu no estudo de Yu et al. $2020^{19}$, 
onde de três RNs testados para a COVID-19, um neonato apresentou contaminação após 36 horas do nascimento, assim, Zimmermann e Curtis, 202020, acrescentam que a transmissão vertical não pode ser descartada. Deve-se frisar ainda, que novos estudos e pesquisas estão sendo desenvolvidas, com o ímpeto de descobrir se há outros mecanismos que impulsionam a transmissão e a infecção, assim como o seu aspecto de progressão no organismo, originando agravos, sequelas e até mesmo consequências fatais ${ }^{20}$.

Os achados de Chen $\mathrm{H}$, et al. $2020^{16}$ sugerem que não há indícios do SARSCoV-2 no leite materno, tornando-se assim, um dado de extrema relevância para o contexto da saúde, pois dessa forma, os profissionais poderão incentivar o aleitamento materno (AM), com o objetivo de promover a aquisição de anticorpos ao $\mathrm{RN}$, desde que haja os devidos cuidados na realização desta prática 14,17,21,22. No entanto, o AM requer muita atenção devido a possibilidade de infectar o neonato durante o processo de amamentação, por gotículas e fluidificações nasais e orais, assim, medidas de proteção devem ser implementadas visando garantir a segurança da mãe e do neonato ${ }^{14,17,21,22}$.

A COVID-19 pode causar complicações como o sofrimento fetal, aborto espontâneo, dificuldade respiratória e parto prematuro ${ }^{13,15,23}$. Ao avaliar 41 grávidas infectadas pelo vírus, foi observado os seguintes parâmetros: taxa de aborto de $39,1 \%$, nascimento prematuro $<37$ semanas de $24,3 \%$, ruptura prematura de membranas ocorreu em $20,7 \%$, pré-eclâmpsia atingiu cerca de $16,2 \%$, enquanto que a restrição ao crescimento intrauterino (RCIU) atingiu 11,7\%, além disso, a taxa de morte perinatal foi de 7,0\%. Dos RNs nascidos vivos, nenhum apresentou indícios de contaminação. Frente a esses resultados, observa-se a importância de um pré-natal precoce com o intuito de minimizar esses agravos, assim, é essencial a inserção de profissionais qualificados no âmbito da saúde, visando atuar de forma significativa na preservação da saúde da mãe e do feto.

De acordo com o estudo conduzido por Yan et al. ${ }^{24}$, dentre 108 gestantes avaliadas, 104 apresentaram achados radiológicos alterados. De 99 pacientes, observou-se que 21 tiveram parto prematuro e, que destas, seis apresentaram ruptura prematura de membrana, destaca-se ainda, que de oito pacientes que estavam no primeiro e segundo trimestre de gestação, um apresentou aborto espontâneo. Segundo Yang et al. ${ }^{25}$ quando a gestante adquiri a infecção no decorrer do primeiro ou segundo trimestre de gestação, não há dados em relação as 
complicações perinatais e fetais, como anomalias congênitas, aborto, parto prematuro espontâneo ou crescimento fetal restrito ${ }^{26}$ complementam que, quando a contaminação pelo vírus ocorre no terceiro trimestre de gestação, é provável que haja ruptura prematura das membranas, parto prematuro, taquicardia e sofrimento fetal. E, por conseguinte, aumentando a chances da transmissão vertical. Segundo o estudo de Shanes et al. $202{ }^{27}$, dentre 16 placentas examinadas, as do terceiro trimestre apresentam uma probabilidade maior de apresentar características como má perfusão vascular materna, vasos maternos lesionados ou alterados, e trombos intervilares. Frente as alterações imunológicas, decorrentes do desenvolvimento embrionário, é notável que durante o último trimestre de gestação, a mulher vivencia um período de importância significativa para a conclusão do desenvolvimento fetal e preparação da evolução para o parto natural ${ }^{27}$.

Wong et al. $2020{ }^{4}$ em seu estudo sobre resultados perinatais do SARS-CoV, em 12 gestantes participantes, dois se recuperaram sem necessidade de intervenção precoce, mas apresentaram complicações quanto a RCIU no decorrer do tratamento, além disso, em um caso houve parto prematuro espontâneo e em outro, cesárea de emergência. A monitorização de vigilância fetal, através de velocimetria arterial umbilical com Doppler e a cardiotocografia diária (CTG) pré-natal, demonstrou resultados satisfatórios ${ }^{4}$. A RCIU pode ser devido a uma patologia respiratória materna, afetando o suprimento de $\mathrm{O} 2$ do feto ou quando há uma patologia debilitante materna, sendo as duas consideradas graves. No caso da COVID-19, também há a RCIU, mas não há estudos que apontem o motivo ${ }^{23,28-31 . ~}$

A indicação de parto precoce foi aderida devido a deterioração materna, déficit na manutenção de oxigênio, dificuldade na implementação da ventilação mecânica (VM) decorrente do útero gravídico, além do comprometimento fetal e outros fatores obstétricos ${ }^{4,13,14,32,33}$. Frente a isso, é de grande importância que os profissionais estejam atentos aos sintomas mais presentes em grávidas, assim como a febre, mialgia, mal estar, calafrios, dor de garganta, rigores, tosse e falta de $\operatorname{ar}^{4,13,14,32,33}$. Além disso, é preponderante destacar que a CTG, ultrassonografia sérica, avaliação do volume de licor e do fluxo sanguíneo, devem ser realizados continuamente, visando identificar alterações ${ }^{4}$. Desse modo, tais mecanismos tornam-se imprescindíveis para mediar a patogenicidade desse vírus no organismo do feto e ministrar a ação mais viável a ser aplicada sobre cada intercorrência ${ }^{24}$.

Em um estudo realizado por Liu et al. $2020^{9}$, o escore do Apgar apresentado 
em nove RNs no primeiro minuto, foi de 10, em outros estudos diferentes envolvendo crianças, neonatos e lactantes, evidenciou-se que o índice foi de 8,8 entre 1 a 5 minutos, respectivamente 21,34 . Outros grupos demostraram que o escore do Apgar em neonatos variou de sete a 10 , durante o $1^{\circ}$ e $5^{\circ}$ minuto ${ }^{11,25,33}$. Ainda, no estudo conduzido por Chen $\mathrm{H}$, et al. ${ }^{(16)}$, dentre nove nascidos vivos, o escore Apgar apresentado em um minuto foi de 8-9 e em 5 minutos, foi de 9-10, além disso, houve monitoramento de sofrimento fetal em dois casos. De acordo com os dados apresentados, observa-se uma boa vitalidade, adaptação e maturidade dos RNs, não apresentando anormalidades que coloquem em risco a integridade de sua saúde, todavia, é relevante acrescentar a importância da implementação de cuidados assistenciais materno infantis, com o ímpeto de evitar a contaminação do RN após o processo de parto e evitar consequências deletérias em seu desenvolvimento ${ }^{11,25,33}$.

\section{Manejo e condutas}

As gestantes precisam manter as mesmas precauções que o restante da população, afim de evitar a infecção por esse vírus, ainda, mesmo que infectadas, devem permanecer sob acompanhamento feito pela equipe de saúde. Quanto a testagem e elegibilidade, o protocolo varia conforme o local em que residem, mas é enfatizado pela OMS, que gestantes sintomáticas para COVID-19, sejam priorizadas para os testes. Visto tais iniciativas, é necessário a implementação e a efetivação de novas políticas de promoção, visando assegurar a integridade assistencial desse grupo de risco ${ }^{35}$. Salienta-se também, um déficit em relação ao atendimento e acolhimento, além da escassez de conhecimento sobre a gravidade desse vírus em mulheres grávidas ${ }^{35}$.

A partir do momento que a gestante é definida como positiva para COVID-19, medidas e estratégias devem ser desenvolvidas e implementadas para assegurar a saúde do binômio mãe e filho. Dessa forma, os principais seguimentos a serem fornecidos e aplicados a uma paciente positiva, são: promover o isolamento, desenvolver a estratificação de risco de acordo com os achados clínicos e a gravidade, determinar a necessidade da realização do parto prematuro, orientar quanto a necessidade de sono e repouso, desenvolver uma nutrição adequada, promover suporte de oxigênio caso seja necessário, atentar-se para a ingesta e perca de líquidos e eletrólitos, avaliar continuamente sinais vitais, saturação, evolução da gestação e batimentos cardiofetais, bem como, desenvolver um planejamento 
assistencial e multiprofissional acerca do parto e promover apoio psicológico ${ }^{7,25,26,36-}$ 38.

Em caso de gestante assintomática ou positiva para COVID-19, que entre em trabalho de parto, as condutas e manejos a serem elencados e priorizados são a adoção de medidas preventivas e de cautela, quanto ao empurrão do feto e a utilização de máscaras cirúrgicas por parte do profissional, com o intuito de evitar uma infecção cruzada ${ }^{25}$. Visto que não há indícios de infecção intrauterina, os cuidados no momento do parto, devem ser redobrados. Contudo, é perceptível a singularidade embargada na relação da prestação de cuidados pactuados a melhor adesão terapêutica. Cabe mencionar a participação do profissional como educador e provedor de políticas de saúde, visando a segurança de todos de forma geral e singular, aderindo-se aos cuidados básicos para atenuar os agravos dessa doença e retroceder a velocidade da sua disseminação 25.

Em relação a amamentação, mesmo que não haja indícios de transmissão pelo $\mathrm{AM}$, as puérperas devem seguir alguns cuidados como a prática da etiqueta respiratória, fazendo uso da máscara de proteção, que deverá ser trocada em caso de espirro, tosse e a cada nova mamada, ainda, higienizar as mãos antes e após mexer com o bebê e desinfetar rotineiramente superfícies que tenham sido tocadas $^{35,39}$. Caso encontre-se debilitada para amamentar, a mulher deve receber apoio, podendo optar por extração do leite, relactação ou até mesmo doação de leite humano. Caso haja sintomas da síndrome gripal, orienta-se o distanciamento mínimo de um metro entre o leito da mãe e o berço do $\mathrm{RN}^{35,39}$. É notável que o binômio mãe e filho vivenciam a atual pandemia de forma mais prejudicial e restrita, afetando diretamente a saúde de ambos. Decorrente disso, torna-se relevante dispor de medidas que fortaleçam o vínculo materno-infantil, evidenciando os conceitos atrelados aos cuidados e as ações mais viáveis a serem designadas, ademais, é imprescindível atentar-se aos princípios básicos que a equipe multiprofissional tem que desenvolver e dominar, diante desse malefício tão complexo e ainda tão desconhecido 35,39 .

\section{Óbito materno: Cenário brasileiro}

Estudos iniciais sobre a COVID-19 no Brasil, traziam informações de que gestantes não apresentavam riscos maiores para o vírus, comparadas com as mulheres não gestantes, diferente da SARS-CoV e MERS-CoV, onde o primeiro 
grupo demonstra pior prognóstico, visto que essas epidemias trouxeram complicações graves para a gravidez, entretanto, novas atualizações veem mostrando o quão grave e prejudicial esse vírus pode ser à saúde $7,16,36,40,41$. Os possíveis agravos podem ser decorrentes da imunodeficiência agregada às adaptações fisiológicas, assim como as respostas orgânicas advindas de infecções pelo vírus. Além disso, é visível que gestantes e puérperas possuem uma desvantagem, pois não há política universal de testes no Brasil, sendo testadas apenas aquelas que já estão apresentando sintomas graves ${ }^{41}$. Visto isso, é de suma relevância que gestantes acima de 28 semanas, cumpram o isolamento social e exerçam as medidas de prevenção, visando evitar as possíveis consequências ${ }^{41}$.

No estudo conduzido por Takemoto et al. 2020 41, durante o período de fevereiro a junho de 2020, com 978 gestantes e puérperas analisadas, diagnosticadas com a Síndrome do Desconforto Respiratório Agudo causada pelo vírus, observouse 124 óbitos, número 3,4 vezes maior (12.7\%), quando comparado as mortes maternas no mundo todo 42,43 , assim, a quantidade de óbitos maternos pelo vírus, representou cerca de $10 \%$ das mortes maternas anuais ocorridas no Brasil, observouse também que a prevalência deste fato foi maior no pós-parto. É visível que a taxa de óbito materno no Brasil é elevada, com isso, profissionais devem estar mais atentos a esse grupo e cientes da gravidade desse vírus ${ }^{41-43}$.

Takemoto et al. $2020^{41}$ sugere como possível causa para essa elevada taxa de óbitos no Brasil, a influência de problemas crônicos no atendimento obstétrico, afetando a saúde materna e perinatal, como exemplo, pode-se citar a má qualidade do pré-natal, violências e intercorrências obstétricas, recursos insuficientes no gerenciamento de cuidados críticos e emergenciais, o acesso desigual aos serviços já existentes intensificou-se com a chegada da pandemia, tornando-se uma barreira adicional, dificultando o acesso aos cuidados, ainda, além do país apresentar uma das maiores taxas de cesariana, há dúvidas quanto ao aumento de risco por morbimortalidade no pós operatório ${ }^{41}$.

Ainda, Rasmussen, Jamieson e Bresee, $2020^{44}$ destacam que em uma pandemia, a gestante apresenta certa resistência em seguir recomendações da unidade de saúde, devido às incertezas dos efeitos colaterais de uma vacina no desenvolvimento do feto ou pela sua exposição a susceptíveis contaminações, durante a consulta de rotina do pré-natal. Assim, nota-se a importância de promover campanhas por parte dos profissionais, com o ímpeto de desmistificar conceitos 
impostos pela sociedade e desenvolver uma autoconscientização visando atender as demandas das gestantes e garantindo-Ihes seus respectivos direitos ${ }^{44}$.

Diante dos dados alarmantes de morte materna no país, houve a criação de um protocolo nacional de atenção as gestantes com COVID-19, dando suporte a uma estratégia oficial unificada que implementa ações a serem realizadas em gestantes, puérperas e em seus respectivos RNs, com o ímpeto de promover bem estar, segurança, diminuição de agravos e sequelas, e posteriormente, redução da morbimortalidade ${ }^{39,45}$. O protocolo abrange desde a atenção básica até as redes de maior complexidade, fornecendo todos os subsídios necessários. O protocolo estabelece que as unidades básicas de saúde, orientem e garantam isolamento às gestantes e as puérperas suspeitas ou confirmadas para COVID-19, e ainda, que as monitorem a cada 24 horas. Quanto às unidades de pronto atendimento, as pacientes que forem submetidas ao internamento, deverão passar por triagem e, posteriormente, serem encaminhadas para locais de média ou alta complexidade, onde há suporte de atendimento mais complexo e avançado. Ressalta-se a importância da notificação compulsória em casos suspeitos de COVID-19, seja em instituições públicas ou privadas, notificando a unidade de vigilância epidemiológica ou órgão correspondente ${ }^{45}$.

Com o intuito de reduzir as idas aos serviços de saúde e o risco de exposição ao vírus, devem ser realizadas tele consultas na $11^{\underline{a}}$ semana, $16-18^{\underline{a}}$ semana, na $32^{\underline{a}}$ e 38 a semana, e posterior a alta hospitalar. Caso não seja possível, as consultas presenciais deverão concentrar-se nas 11, 20, 28, 32, 35, 37, 39 semanas, após, semanalmente até o parto. Não menos importante, o local de atendimento deverá ser isolado, longe de pacientes com sintomas respiratórios ${ }^{45}$.

Para pacientes positivas, deve-se prestar atenção a aspectos emocionais, como estresse pós-traumático ou ansiedade. Quando a gestante possui comorbidades presentes, como diabetes, obesidade, cardiopatia, entre outras, essa se encaixa no grupo de alto risco obstétrico-neonatal, ainda, para casos moderados e graves, recomenda-se a realização dos seguintes exames: hemograma, sódio, creatinina, uréia, potássio, transaminases, LDH, PCR, TP, D-Dímeros, TTPa, ferritina, gasometria arterial e $\mathrm{Rx}$ de tórax ${ }^{45}$.

Em relação a vigilância fetal, esta deve ser realizada afim de avaliar o crescimento fetal, a insuficiência placentária e o oligoâmnio, bem como, é indicada a realização de ultrassonografia mensalmente. Quando houver internação da gestante, 
a vitalidade vital deverá ser estudada diariamente com objetivo de constatar anormalidades ou desfechos aleatórios que almejam cuidados direcionados e de rápida intervenção ${ }^{45}$.

\section{CONCLUSÃO}

Levando em consideração os aspectos observados, nota-se a importância de compreender a ação do SARS-CoV-2 e as possíveis complicações desencadeadas por esse vírus no organismo da gestante, visto que se tornou um problema de saúde em nível mundial.

Não há dados suficientes que promovam uma confirmação verídica da transmissão vertical, transplacentária ou pela amamentação do vírus SARS-CoV-2, no entanto, há indícios da existência de uma provável transmissão transplacentária, além disso, mesmo se essa possibilidade for confirmada, ela poderá ser caracterizada como não frequente por apresentar baixas taxas de incidência, assim, torna-se relevante a observação e elaboração de cuidados assistenciais prestados ao binômio mãe e filho, por intermédio da inserção de protocolos nacionais e adesão de cuidados básicos pertinentes à prevenção.

Percebe-se também, que são escassos os estudos publicados e que existem muitas dúvidas pertinentes ao potencial de contaminação, agravos e sequelas do mesmo. Desse modo, é fundamental a realização de estudos que englobem essa temática com o teor de fornecer novos esclarecimentos e conhecimento, sobre a interação do SARS-CoV-2 durante o período gestacional, puerperal e no desenvolvimento dos recém-nascidos.

\section{REFERÊNCIAS}

1. Borges do Nascimento IJ, Cacic N, Abdulazeem HM, von Groote TC, Jayarajah U, Weerasekara I, et al. Novel Coronavirus Infection (COVID-19) in Humans: A Scoping Review and Meta-Analysis. J Clin Med. [Internet]. 2020 [acesso em 2020 ago 06];9(4):941. doi: https://doi.org/10.3390/jcm9040941

2. De Oliveira Lima CMA. Information about the new coronavirus disease (COVID19). Radiol Bras. [Internet]. 2020 [acesso em 2020 ago 13];53(2):5. doi: https://doi.org/10.1590/0100-3984.2020.53.2e1 
3. Chate RC, Ururahy EK, Fonseca N, Bastos R, Passos D, Borges Da G, et al. Presentation of pulmonary infection on CT in COVID-19: initial experience in Brazil. 2020 J. bras. pneumol. [Internet]. 2020 [acesso em 2020 ago 06];46(2). doi: https://doi.org/10.36416/1806-3756/e20200121

4. Wong SF, Chow KM, Leung TN, Ng WF, Ng TK, Shek CC, et al. Pregnancy and perinatal outcomes of women with severe acute respiratory syndrome. Am J Obstet Gynecol. 2004 [Internet]. 2020 [acesso em 2020 ago 13 ];191(1):292-7. doi: https://doi.org/10.1016/j.ajog.2003.11.019

5. Croda J, Oliveira WK de, Frutuoso RL, Mandetta LH, Baia-Da-silva DC, BritoSousa JD, et al. Covid-19 in Brazil: Advantages of a socialized unified health system and preparation to contain cases. Rev Soc Bras Med Trop. 2020 [Internet]. 2020 [acesso em 2020 ago 20];53. doi: https://doi.org/10.1590/0037-8682-01672020

6. Alfaraj SH, Al-Tawfiq JA, Memish ZA. Middle East Respiratory Syndrome Coronavirus (MERS-CoV) infection during pregnancy: Report of two cases \& review of the literature. J Microbiol Immunol Infect. [Internet]. 2019 [acesso em 2020 ago 27];52(3): 501-503. doi: https://doi.org/10.1016/i.jmii.2018.04.005

7. Rasmussen SA, Smulian JC, Lednicky JA, Wen TS, Jamieson DJ. Coronavirus Disease 2019 (COVID-19) and pregnancy: what obstetricians need to know. Am J Obstet Gynecol. [Internet]. 2020 [acesso em 2020 ago 20];222(5):415-426. doi: https://doi.org/10.1016/j.ajog.2020.02.017

8. Schwartz DA. An analysis of 38 pregnant women with COVID-19, their newborn infants, and maternal-fetal transmission of SARS-CoV-2: Maternal coronavirus infections and pregnancy outcomes. Arch Pathol Lab Med. [Internet]. 2020 [acesso em 2020 ago 20];144 (7): 799-805. doi: https://doi.org/10.5858/arpa.2020-0901$\underline{S A}$

9. Liu Y, Chen H, Tang K, Guo Y. Clinical manifestations and outcome of SARS-CoV2 infection during pregnancy. J Infect. [Internet]. 2020 [acesso em 2020 ago 20]. doi: https://doi.org/10.1016/j.jinf.2020.02.028

10. Karimi-Zarchi M, Neamatzadeh H, Dastgheib SA, Abbasi H, Mirjalili SR, Behforouz A, et al. Vertical Transmission of Coronavirus Disease 19 (COVID-19) from Infected Pregnant Mothers to Neonates: A Review. Fetal Pediatr Pathol. [Internet]. 2020 [acesso em 2020 ago 20];39(3):246-250. doi: https://doi.org/10.1080/15513815.2020.1747120

11. Kasraeian M, Zare M, Vafaei H, Asadi N, Faraji A, Bazrafshan K, et al. COVID-19 pneumonia and pregnancy; a systematic review and meta-analysis. J Matern Fetal Neonatal Med. [Internet]. 2020 [acesso em 2020 ago 20];19;1-8. doi: https://doi.org/10.1080/14767058.2020.1763952 
12. Peyronnet V, Sibiude J, Deruelle P, Huissoud C, Lescure X, Lucet JC, et al. SARSCoV-2 infection during pregnancy. Information and proposal of management care. Gynecol Obstet Fertil Senol. [Internet]. 2020 [acesso em 2020 ago 27];48(5):436443. doi: https://doi.org/10.1016/i.gofs.2020.03.014

13. Dana PM, Kolahdooz F, Sadoughi F, Moazzami B, Chaichian S, Asemi Z. COVID19 and pregnancy: A Review of current knowledge. Infez Med. [Internet]. 2020 [acesso em 2020 ago 27];1(28)46-51. Disponível em: https://pubmed.ncbi.nlm.nih.gov/32532938/

14. Pereira A, Cruz-Melguizo S, Adrien M, Fuentes L, Marin E, Perez-Medina T. Clinical course of coronavirus disease-2019 in pregnancy. Acta Obstet Gynecol Scand. [Internet]. 2020 [acesso em 2020 ago 27];99(7):839-847. doi: https://doi.org/10.1111/aogs.13921

15. Panahi L, Amiri M, Pouy S. Clinical Characteristics of COVID-19 Infection in Newborns and Pediatrics: A Systematic Review. Arch Acad Emerg Med. [Internet]. 2020 [acesso em 2020 ago 27];18;8(1):e50. Disponível em: https://www.ncbi.nlm.nih.gov/pmc/articles/PMC7212072

16. Chen H, Guo J, Wang C, Luo F, Yu X, Zhang W, et al. Clinical characteristics and intrauterine vertical transmission potential of COVID-19 infection in nine pregnant women: a retrospective review of medical records. Lancet. [Internet]. 2020 [acesso em 2020 ago 27];395(10226):809-815. doi: https://doi.org/10.1016/S0140$\underline{6736(20) 30360-3}$

17. Rajewska A, Mikołajek-Bedner W, Lebdowicz-Knul J, Sokołowska M, Kwiatkowski S, Torbé A. COVID-19 and pregnancy-where are we now? A review. J Perinat Med. [Internet]. 2020 [acesso em 2020 ago 27];48(5):428-434. doi: https://doi.org/10.1515/jpm-2020-0132

18. Elshafeey F, Magdi R, Hindi N, Elshebiny M, Farrag N, Mahdy S, et al. A systematic scoping review of COVID-19 during pregnancy and childbirth. Int $\mathrm{J}$ Gynaecol Obstet. [Internet]. 2020 [acesso em 2020 ago 27];150(1):47-52. doi: https://doi.org/10.1002/ijgo.13182

19. Yu N, Li W, Kang Q, Xiong Z, Wang S, Lin X, et al. Clinical features and obstetric and neonatal outcomes of pregnant patients with COVID-19 in Wuhan, China: a retrospective, single-centre, descriptive study. Lancet Infect Dis. [Internet]. 2020 [acesso em 2020 ago 13];20(5):559-564. doi: https://doi.org/10.1016/S1473$\underline{3099(20) 30176-6}$

20. Zimmermann P, Curtis N. COVID-19 in Children, Pregnancy and Neonates: A Review of Epidemiologic and Clinical Features. Pediatr Infect Dis J. [Internet]. 2020 [acesso em 2020 ago 13];39(6):469-477. doi: https://doi.org/10.1097/INF.0000000000002700 
21. Martins-Filho PR, Santos VS, Santos HP. To breastfeed or not to breastfeed? Lack of evidence on the presence of SARS-CoV-2 in breastmilk of pregnant women with COVID-19. Rev Panam Salud Publica. [Internet]. 2020 [acesso em 2020 ago 132];44:e59. doi: https://doi.org/10.26633/RPSP.2020.59

22. Lowe B, Bopp B. COVID-19 vaginal delivery - A case report. Aust N Z J Obstet Gynaecol. [Internet]. 2020 [acesso em 2020 ago 13];60(3):465-466. doi: https://doi.org/10.1111/ajo.13173

23. Di Mascio D, Khalil A, Saccone G, Rizzo G, Buca D, Liberati M, et al. Outcome of coronavirus spectrum infections (SARS, MERS, COVID-19) during pregnancy: a systematic review and meta-analysis. Am J Obstet Gynecol MFM. [Internet]. 2020 [acesso em 2020 ago 13];2(2):100107. doi: https://doi.org/10.1016/..ajogmf.2020.100107

24. Yan J, Guo J, Fan C, Juan J, Yu X, Li J, et al. Coronavirus disease 2019 in pregnant women: a report based on 116 cases. Am J Obstet Gynecol. [Internet]. 2020 [acesso em 2020 ago 13];223(1):111.e1-111.e14. doi: https://doi.org/10.1016/j.ajog.2020.04.014

25. Yang $\mathrm{H}$, Wang $\mathrm{C}$, Poon LC. Novel coronavirus infection and pregnancy. Ultrasound Obstet Gynecol. [Internet]. 2020 [acesso em 2020 ago 13]; 55(4):435437. doi: https://doi.org/10.1002/uog.22006

26. Liang H, Acharya G. Novel corona virus disease (COVID-19) in pregnancy: What clinical recommendations to follow? Acta Obstet Gynecol Scand. [Internet]. 2020 [acesso em 2020 ago 13];99(4):439-442. doi: https://doi.org/10.1111/aogs.13836

27. Shanes ED, Mithal LB, Otero S, Azad HA, Miller ES, Goldstein JA. Placental Pathology in COVID-19. Am J Clin Pathol. [Internet]. 2020 [acesso em 2020 ago 13];154(1):23-32. doi: https://doi.org/10.1093/ajcp/aqaa089

28. Rondelli G, Jardim D, Hamad G, Luna E, Marinho W, Mendes L, et al. Assistência às gestantes e recém-nascidos no contexto da infecção Covid-19: Uma Revisão Sistemática. DESAFIOS - Rev Interdiscip da Univ Fed do Tocantins. [Internet]. 2020 [acesso em 2020 ago 06];7(Especial-3), 48-74. doi: https://doi.org/10.20873/uftsuple2020-8943

29. de Sousa ÁFL, de Carvalho HEF, de Oliveira LB, Schneider G, Camargo ELS, Watanabe $E$, et al. Effects of COVID-19 infection during pregnancy and neonatal prognosis: What is the evidence?. Int J Environ Res Public Health. [Internet]. 2020 [acesso em 2020 ago 06];17(11):4176. doi: https://doi.org/10.3390/ijerph17114176

30. Qiao J. What are the risks of COVID-19 infection in pregnant women? The Lancet. 2020. Lancet. [Internet]. 2020 [acesso em 2020 ago 06]; 395(10226):760-762. doi: https://doi.org/10.1016/S0140-6736(20)30365-2 
31. Rocha ALL. Contracepção em tempos de Covid-19. SOGIMIG. [Internet]. 2020 [acesso em 2020 ago 06]. Disponível em: https://www.sogimig.org.br/wpcontent/uploads/2020/04/Sogimig Covid-19 Contracep\%C3\%A7ao Paramedicos Abr-2020.pdf

32. Tsang OTY, Chau TN, Choi KW, Tso EYK, Lim W, Chiu MC, et al. Coronaviruspositive Nasopharyngeal Aspirate as Predictor for Severe Acute Respiratory Syndrome Mortality. Emerg Infect Dis. [Internet]. 2003 [acesso em 2020 ago 06]; 9(11):1381-7. doi: https://doi.org/10.3201/eid0911.030400

33. Juan J, Gil MM, Rong Z, Zhang Y, Yang H, Poon LC. Effect of coronavirus disease 2019 (COVID-19) on maternal, perinatal and neonatal outcome: systematic review. Ultrasound Obstet Gynecol. [Internet]. 2020 [acesso em 2020 ago 06];56(1):1527. doi: https://doi.org/10.1002/uog.22088

34. Panahi L, Amiri M, Pouy S. Risks of Novel Coronavirus Disease (COVID-19) in Pregnancy; a Narrative Review. Arch Acad Emerg Med. [Internet]. 2020 [acesso em 2020 ago 26];8(1):e34. Disponível em: https://pubmed.ncbi.nlm.nih.gov/32232217/

35. Secretaria de Saúde de Tocantins. Atenção à Gestante - Coronavírus (Covid-19) [Internet].Palmas; 2020. [citado em 2018 Dez 29]. Disponível em: https://www.to.gov.br/saude/atencao-a-gestante-coronavirus-covid19/6itpcs6jdi00

36. Chen D, Yang H, Cao Y, Cheng W, Duan T, Fan C, et al. Expert consensus for managing pregnant women and neonates born to mothers with suspected or confirmed novel coronavirus (COVID-19) infection. Int J Gynaecol Obstet. [Internet]. 2020 [acesso em 2020 ago 26];149(2):130-136. doi: https://doi.org/10.1002/ijgo.13146

37. Chua MSQ, Lee JCS, Sulaiman S, Tan HK. From the frontline of COVID-19 - how prepared are we as obstetricians? A commentary. BJOG. [Internet]. 2020 [acesso em 2020 ago 26];127(7):786-788. doi: https://doi.org/10.1111/1471-0528.16192

38. Dashraath P, Wong JLJ, Lim MXK, Lim LM, Li S, Biswas A, et al. Coronavirus disease 2019 (COVID-19) pandemic and pregnancy. Am J Obstet Gynecol. [Internet]. 2020 [acesso em 2020 ago 26];222(6):521-531. doi: https://doi.org/10.1016/j.ajog.2020.03.021

39. Ministério da Saúde. Nota técnica no 6/2020-COCAM/CGCIVI/DAPES/SAPS/MS: Atenção à saúde do recém-nascido no contexto da Infecção do novo coronavírus (SARS-CoV-2) [Internet]. Brasília; 2020. [citado em 2018 nov 26].Disponível em: http://www.crn2.org.br/crn2/conteudo/nt 6.pdf

40. De Seixas Filho JT, Seyfarth MSC, Cunha DM, Da Silveira GRRA, Guedes EF, Orsini M, et al. Recomendações de prevenção da saúde materno-infantil na pandemia COVID-19 por meio de protocolos médicos. Rev Augustus. [Internet]. 
2020 [acesso em 2020 ago 26];25(51). doi: https://doi.org/10.15202/1981896.2020v25n51p316

41. Takemoto MLS, Menezes M de O, Andreucci CB, Nakamura-Pereira M, Amorim MMR, Katz L, et al. The tragedy of COVID-19 in Brazil: 124 maternal deaths and counting. Int J Gynaecol Obstet. [Internet]. 2020 [acesso em 2020 nov 06]; 151(1):154-156. doi: https://doi.org/10.1002/ijgo.13300

42. Knight M, Bunch K, Vousden N, Morris E, Simpson N, Gale C, et al. Characteristics and outcomes of pregnant women admitted to hospital with confirmed SARS-CoV2 infection in UK: National population based cohort study. BMJ. [Internet]. 2020 [acesso em 2020 ago 26];369:m2107. doi: https://doi.org/10.1136/bmj.m2107

43. Ellington S, Strid P, Tong VT, Woodworth K, Galang RR, Zambrano LD, et al. Characteristics of Women of Reproductive Age with Laboratory-Confirmed SARSCoV-2 Infection by Pregnancy Status. Morb Mortal Wkly Rep. [Internet]. 2020 [acesso em 2020 ago 26];69(25):769-775. doi: https://doi.org/10.15585/mmwr.mm6925a1

44. Rasmussen SA, Jamieson DJ, Bresee JS. Pandemic influenza and pregnant women. Emerg Infect Dis. [Internet]. 2008 [acesso em 2020 ago 26];14(1):95-100. doi: https://doi.org/10.3201/eid1401.070667

45. Ministério da Saúde. Assistência à gestante e puérpera frente à pandemia de COVID-19 assistência à gestante e puérpera frente à pandemia de COVID19.[Internet]. Brasília; 2020. [citado em 2020 agosto 06]. Disponível em: http://189.28.128.100/dab/docs/portaldab/documentos/corona/manual recomend acoes gestantes covid19.pdf

46. Weffort VRS, Rodrigues BR, Prado EO; Calapodopulos NVI, Silva KCBK, Cunal VCA. Transmissão vertical da covid-19: uma revisão integrativa. Revista Pediátrica. [Internet]. 2020 [acesso em 2021 out 11]. Disponível em: https://cdn.publisher.gn1.link/residenciapediatrica.com.br/pdf/rp200820a01.pdf

47. Fundação Oswaldo Cruz. O Boletim do Observatório Covid-19 Fiocruz. [Internet]. Rio de Janeiro; 2021. [citado em 2021 outubro 11] Disponível em: https://agencia.fiocruz.br/sites/agencia.fiocruz.br/files/u34/boletim covid 2021semanas 20-21-red.pdf 